\title{
Diuretic, Natriuretic, and Vasodepressor Activity of a Lipid Fraction Enhanced in Medium of Cultured Mouse Medullary Interstitial Cells by a Selective Fatty Acid Amide Hydrolase Inhibitor
}

\author{
Zdravka Daneva, ${ }^{1}$ Sara K. Dempsey, ${ }^{1}$ Ashfaq Ahmad, Ningjun Li, Pin-Lan Li, \\ and Joseph K. Ritter \\ Department of Pharmacology and Toxicology, Virginia Commonwealth University School of Medicine, Richmond, Virginia \\ Received July 26, 2018; accepted December 4, 2018
}

\begin{abstract}
The relationship between the endocannabinoid system in the renal medulla and the long-term regulation of blood pressure is not yet understood. To investigate the possible role of the endocannabinoid system in renomedullary interstitial cells, mouse medullary interstitial cells (MMICs) were obtained, cultured, and characterized for their responses to treatment with a selective inhibitor of fatty acid amide hydrolase, PF-3845 (N-3-pyridinyl-4-[[3-[[5-(trifluoromethyl)-2pyridinyl] [oxy]pheny[]methyl]-1-piperidinecarboxamide). Treatment of MMICs with PF-3845 increased cytoplasmic lipid granules detected by Sudan Black B staining and multilamellar bodies identified by transmission electron microscopy. High-performance liquid chromatography (HPLC) analyses of lipid extracts of MMIC culture medium revealed a 205-nm absorbing peak that showed responsiveness to PF-3845 treatment. The biologic activities of the PF-3845-induced product (PIP) isolated by HPLC were investigated
\end{abstract}

in anesthetized, normotensive surgically instrumented mice. Intramedullary and intravenous infusion of PIP at low dose rates (0.5-1 area units under the peak/10 $\mathrm{min}$ ) stimulated diuresis and natriuresis, whereas these parameters returned toward baseline at higher doses but mean arterial pressure (MAP) was lowered. Whereas intravenous bolus doses of PIP stimulated diuresis, the glomerular filtration rate, and medullary blood flow (MBF) and reduced or had no effect on MAP, an intraperitoneal bolus injection of PIP reduced MAP, increased MBF, and had no effect on urine parameters. These data support a model whereby PF-3845 treatment of MMICs results in increased secretion of a neutral lipid that acts directly to promote diuresis and natriuresis and indirectly through metabolites to produce vasodepression. Efforts to identify the structure of the PF-3845-induced lipid and its relationship to the previously proposed renomedullary antihypertensive lipids are ongoing.

\section{Introduction}

The kidneys are considered critical for the long-term control of blood pressure by regulation of body fluid and blood volume. In support of this concept, the kidneys control the systemic

This work was supported by the National Institutes of Health National Institute of Diabetes and Digestive and Kidney Diseases [Grant R01DK102539] and the National Institutes of Health National Institute on Drug Abuse [Grant P30DA033934 and Training Grant T32DA007027 (to S.K.D.)]. Transmission electron microscopy was performed at the Virginia Commonwealth University Microscopy Facility, which is supported in part by funding from the National Institutes of Health National Cancer Institute [Cancer Center Support Grant P30CA016059].

This work was previously presented in part Zdravka Daneva, Sara Dempsey, Ashfaq Ahmad, Ningjun Li, Pin-Lan Li, and Joseph Ritter. Diuretic and natriuretic activity of a lipid induced by fatty acyl amide hydrolase inhibition in cultured mouse medullary interstitial cells. Experimental Biology 2017; 2017 April 20-24; Chicago, IL.

${ }^{1}$ Z.D. and S.K.D. contributed equally to this work.

https://doi.org/10.1124/jpet.118.252320. activity of the prohypertensive renin-angiotensin-aldosterone system by regulating the production and release of renin from juxtaglomerular cells in response to decreased renal perfusion pressure or reduced sodium load in the distal tubules (Karlstrom et al., 1990). They have also been proposed to contain a parallel antihypertensive hormonal system, the medullipin system (Muirhead, 1993; Folkow, 2007). In this system, a neutral antihypertensive lipid, known as medullipin or renomedullary neutral antihypertensive lipid, is secreted from interstitial cells of the renal medulla in response to increased medullary perfusion pressure (Gothberg et al., 1982). Medullipin was shown to possess several characteristics consistent with its antihypertensive activity, including vasodepressor activity, inhibition of sympathetic tone, and diuretic effects (Karlstrom et al., 1988). However, attempts to isolate and identify this substance from sources in which it was detected, including extracts of the renal medulla, renal

ABBREVIATIONS: AM4054, 9 $\beta$-(hydroxymethyl)-3-(1-adamantyl)-hexahydrocannabinol; AM7418, butyl-2-[(6aR, 10aR)-6a,7,10,10a-tetrahydro-1hydroxy-9-(hydroxymethyl)-6,6-dimethyl-6H-benzo[c]chromen-3-yl]-2-methylpropanoate; $\mathrm{AU}$, area units under the peak; COX, cyclooxygenase; DMEM, Dulbecco's modified Eagle's medium; FAAH, fatty acid amide hydrolase; FITC, fluorescein isothiocyanate; GFR, glomerular filtration rate; HPLC, high-performance liquid chromatography; HRP, horseradish peroxidase; KO, knockout; MAP, mean arterial pressure; MBF, medullary blood flow; MMIC, mouse medullary interstitial cell; PBS, phosphate-buffered saline; PF-3845, N-3-pyridinyl-4-[[3-[[5-(trifluoromethyl)-2-pyridinyl]oxy]phenyl]methyl]-1-piperidinecarboxamide; PIP, PF-3845-induced product; $\mathrm{U}_{\mathrm{K}}$, potassium excretion rate; $\mathrm{U}_{\mathrm{Na}}$, sodium excretion rate; WIN55,212-2, (R)-(+)-[2,3-dihydro-5-methyl-3-(4-morpholinylmethyl)pyrrolo[1,2,3-de]-1,4-benzoxazin-6-yl]-1-naphthalenylmethanone mesylate; WT, wild type. 
venous effluents, or medullary interstitial cells, did not prove successful (Brooks et al., 1994; Glodny and Pauli, 2006).

Our group is investigating the hypothesis that anandamide or metabolite(s) of anandamide may be related to the proposed antihypertensive renomedullary substance. Anandamide, the $N$-acyl ethanolamide of arachidonic acid (Devane et al., 1992), is chemically neutral at physiologic $\mathrm{pH}$. Anandamide was first identified as the endogenous cannabinoid receptor agonist (Bermudez-Silva et al., 2010). The kidney, especially the renal medulla, is unique in its high content of anandamide and the primary anandamide-hydrolyzing enzyme, fatty acid amide hydrolase (FAAH) (Long et al., 2011; DiPatrizio and Piomelli, 2012; Ritter et al., 2012). Anandamide has actions in the cardiovascular and renal systems, which are consistent with an antihypertensive role, including vasodepressor activity (Lake et al., 1997), sympatholytic effects (Ishac et al., 1996; Deutsch et al., 1997), and diuretic and natriuretic activities (Li and Wang, 2006). Infusion of exogenous anandamide into the renal medulla stimulated diuresis and natriuresis (Ritter et al., 2012), an effect that was mimicked by intramedullary infusion of known FAAH inhibitors, including isopropyl dodecylfluorophosphate (Ahmad et al., 2017) or PF-3845 ( $N$ 3-pyridinyl-4-[[3-[[5-(trifluoromethyl)-2-pyridinyl]oxy]phenyl]methyl]-1-piperidinecarboxamide) (Ahmad et al., 2018). These studies support a role of endogenous anandamide or other fatty acyl ethanolamides that accumulate upon inhibition of their hydrolysis. However, in the latter studies, the cellular source in the renal medulla of the diuretic and natriuretic lipid ethanolamides remained unknown.

An important nontubular cell type of the renal medulla is the lipid-laden (type I) interstitial cell (Lemley and Kriz, 1991). Characteristics of this type of interstitial cell include its stellate-like cellular morphology with cytoplasmic projections and increased cell density toward the tip of the renal papilla. They are noticeable in stained sections of the renal medulla based on their long axes lying perpendicular to loop of Henle tubules and vasa recta cells, producing a characteristic ladderlike appearance. Although cytoplasmic projections from these cells do not make physical contact with the loop of Henle or vasa recta, they have been suggested to play a role in signaling to these cell types in response to changing conditions (Lemley and Kriz, 1991). Another hallmark is the presence of osmiophilic lipid granules that are rich in arachidonic acid, the precursor of eicosanoid metabolites such as prostaglandin $\mathrm{E}_{2}$. The synthesis of prostaglandin $\mathrm{E}_{2}$ by interstitial cells was stimulated by angiotensin II and other vasoactive peptides and certain physiologic conditions (Zusman and Keiser, 1977a). Changes in the number of these lipid droplets in experimental models of hypertension (Bohman and Jensen, 1976) suggested a role of these lipid droplets in the secretion of medullipin in response to elevated renal perfusion pressure (Muirhead, 1993; Folkow, 2007).

The putative role of interstitial cells in secreting the renomedullary antihypertensive substance led us to isolate these cells from the renal medulla of mice to study their responses to FAAH inhibition. The isolated cells were assessed for histologic, immunohistochemical, and ultrastructural markers consistent with their identity as medullary interstitial cells, and also for the presence of FAAH enzyme. The effect of treating the cells with the selective FAAH inhibitor, PF-3845, on the appearance of lipids in the medium of mouse medullary interstitial cells (MMICs) was investigated. During the investigation, an unknown 205-nm absorbing product was identified in lipid extracts of the medium of cultured MMICs, which showed enhancement by treatment with PF-3845. The 205-nm absorbing substance was purified by high-performance liquid chromatography (HPLC) and was characterized in an acute renal function model using anesthetized mice after different routes of administration.

\section{Materials and Methods}

Reagents. Trypsin-EDTA $(10 \times, 0.5 \%)$, penicillin-streptomycin $(100 \times)$, and glutamine $(100 \times)$ were purchased from GibcoInvitrogen (Gaithersburg, MD). Fetal bovine serum was from Serum Source International (Charlotte, NC). Dulbecco's modified Eagle's medium (DMEM) with high glucose was from Gibco/Life Technologies (Grand Island, NY). Polyclonal IgG antibodies to tenascin C (H-300, sc-20932), cyclooxygenase (COX)-2 (M19, sc-1747), and FAAH (C-20, sc-26428) were from Santa Cruz Biotechnology (Dallas, TX). Sudan Black B was from Santa Cruz Biotechnology. Horseradish peroxidase (HRP)-conjugated anti-rabbit and anti-goat IgG antibodies were from Jackson ImmunoResearch Laboratories (West Grove, PA). 3,3'Diaminobenzidine (ImmPACT DAB Peroxidase Substrate) was from Vector Laboratories (Burlingame, CA). Indomethacin was from CalBiochem (La Jolla, CA) and Sigma Chemical (St. Louis, MO), respectively. PF-3845 was purchased from ApexBio (Houston, TX). HPLC-grade water and methanol were purchased from Thomas Scientific (Swedesboro, NJ). Medical-grade nitrogen was purchased from National Welders Supply Company (Richmond, VA).

Animals. Male C57BL/6J mice (Jackson Laboratory, Bar Harbor, $\mathrm{ME})$ and male and female homozygous $\mathrm{FAAH}^{+/+}$wild-type (FAAH WT) and FAAH $^{-1-}$ knockout (FAAH KO) mice (2-5 months old) (from a colony established by Dr. Aron Lichtman at Virginia Commonwealth University, Richmond, VA) were used in this study. The FAAH KO mouse colony has been maintained by backcrossing onto a C57BL/6 background for more than 20 generations (Wise et al., 2008). The mice in this study weighed 20-35 g and were housed four to five per cage in a temperature-controlled $\left(20-22^{\circ} \mathrm{C}\right)$ and humidity-controlled $(50 \%-$ $55 \%$ ) environment with a 12-hour light/dark cycle with free access to food and water. All experiments involving animals in this study were approved by the Institutional Animal Care and Use Committee of Virginia Commonwealth University in accordance with the National Institutes of Health Guide for the Care and Use of Laboratory Animals.

MMICs. Mouse renomedullary interstitial cells were established in primary culture from transplants of isolated and fragmented mouse renal inner medulla using the approach of Muirhead et al. (1990) and subsequently applied and adapted by many others (Dunn et al., 1976; Zusman and Keiser, 1977b; Zou et al., 2001; Moeckel et al., 2003), including our laboratories (Li et al., 2007; Wang et al., 2011). Briefly, C57BL/6J mice were anesthetized using thiobutabarbital (Inactin; Sigma-Aldrich, St. Louis, MO) and ketamine (Ketathesia; Henry Schein Animal Health, Dublin, $\mathrm{OH}$ ) and sacrificed, and the kidneys were removed aseptically. The kidneys were bisected and the inner medullary regions were excised, minced, and suspended in DMEM. The suspended fragments were injected subcutaneously over the left rear flank of an isogenic recipient mouse using an 18-gauge syringe needle. After 7-10 days, the recipient mouse was anesthetized and sacrificed, and the yellowish nodules attached to the serosal surfaces over the injection sites were excised. The nodules were minced, trypsinized with $0.05 \%$ trypsin-EDTA at $37^{\circ} \mathrm{C}$ for 15 minutes (Gibco/ Life Technologies), and centrifuged at $500 \mathrm{~g}$ for 5 minutes. The pellet was resuspended in DMEM containing $10 \%$ fetal calf serum, $100 \mathrm{U} / \mathrm{ml}$ penicillin $\mathrm{G}$, and $100 \mu \mathrm{g} / \mathrm{ml}$ streptomycin and transferred to a single well of a 12-well tissue culture dish. For the first week after plating, the growth medium also contained amphotericin B $(25 \mu \mathrm{g} / \mathrm{ml})$. At $90 \%$ confluence, the cells were trypsinized and passaged successfully to 
larger dishes. At passage 4, the cells were trypsinized, resuspended in frozen storage medium ( $80 \%$ DMEM, $10 \%$ fetal bovine serum, and 10\% DMSO), and stored in frozen aliquots under liquid nitrogen. MMICs were maintained by regular feeding in complete growth medium, passaging every 7 days (1:3 split ratio). The experiments described in this work used MMICs between passages 4-15.

Transmission Electron Microscopic Analysis. For ultrastructural imaging, cells were trypsinized, pelleted, resuspended in phosphate-buffered saline (PBS), repelleted, and resuspended in $0.1 \mathrm{M}$ cacodylate buffer, $\mathrm{pH} 7$, containing $2.5 \%$ glutaraldehyde at room temperature. Subsequently, the cells were fixed in osmium tetroxide, dehydrated, and embedded according to standard methods. Sections (600-700 A thick mounted on grids) were prepared using a Leica EM UC6i Ultramicrotome (Leica Microsystems, Buffalo Grove, IL) then stained with 5\% uranyl acetate and Reynold's lead citrate. Imaging was performed with a JEOL JEM-1230 transmission electron microscope (Jeol U.S.A. Inc., Peabody, MA) with a Gatan Orius SC1000 digital camera (Gatan, Pleasanton, CA).

Immunohistochemistry of Kidney Tissue and Cultured MMICs. MMICs were cultured on tissue culture dishes overnight and then fixed in $4 \%$ paraformaldehyde in PBS for 15 minutes. For kidney tissue sections, mouse kidneys were perfused in situ with PBS followed by neutral $4 \%$ paraformaldehyde. After excision, the kidneys were fixed overnight in 10\% neutral-buffered formalin and embedded. Tissue sections $(5 \mu \mathrm{m})$ cut onto glass slides were processed for immunostaining as described previously ( $\mathrm{Li}$ et al., 2007). After washing in PBS with $0.1 \%$ Tween 20, the samples were incubated with $3 \%$ blocking serum for 30 minutes at room temperature. Primary antibodies and their dilutions (in PBS with $0.1 \%$ Tween 20) were tenascin C (1:50 dilution), COX-2 M-19 (1:50), and FAAH C-20 (1:50). Secondary antibodies were diluted 1:200. Incubations with primary antibodies were overnight at $4^{\circ} \mathrm{C}$ and with HRP-conjugated secondary antibodies for 30 minutes at room temperature with gentle rocking. HRP staining was detected using 3,3'-diaminobenzidine as a substrate. After counterstaining nuclei with hematoxylin, stained tissues and cells were washed, mounted, and observed by phase-contrast microscopy.

Sudan Black B Lipid Staining. MMICs were stained for neutral lipids using Sudan Black B (Schneider et al., 2015). After washing and fixing as described above, the cells were washed with PBS then with $70 \%$ ethanol, followed by staining for 10 minutes at room temperature with Sudan Black B solution $(0.7 \%$ in $70 \%$ ethanol, filtered immediately before use). Cells were washed with PBS and observed by phasecontrast light microscopy.

Dose Response of PF-3845-Inducible MMIC Product. MMICs $\left(3 \times 10^{6}\right.$ cells per T-175 $\mathrm{cm}^{2}$ flask) were plated and incubated with either vehicle (ethanol, $0.1 \%$ ) or PF-3845 at the indicated concentrations (dissolved in ethanol, $0.1 \%$ final concentration) for 24 hours in a humidified $37^{\circ} \mathrm{C}$ incubator with $5 \% \mathrm{CO}_{2}$. In some cases, the effect of indomethacin or celecoxib (10 $\mu \mathrm{M}$ final) was tested either alone or in combination with PF-3845. Complete growth medium was incubated without cells as a control. Lipids were extracted by the Folch method (Folch et al., 1957). Briefly, culture medium (1 ml) was extracted with a $2: 1(\mathrm{v} / \mathrm{v})$ mixture of chloroform/methanol $(2 \mathrm{ml})$ and $0.73 \%$ sodium chloride $(200 \mu \mathrm{l})$. After centrifugation, the organic phase was collected, and the aqueous phase was extracted twice more with chloroform $(1 \mathrm{ml})$. The organic phases were combined and evaporated to dryness under nitrogen. The residue was reconstituted in $500 \mu \mathrm{l}$ methanol and $50 \mu \mathrm{l}$ was analyzed by reverse phase HPLC with UV detection as described above. The concentration of product was expressed in area units under the peak (AU) normalized for the volume of culture medium.

HPLC Purification of PF-3845-Inducible MMIC Product. MMICs were dissociated by trypsinization and plated in tissue culture dishes $\left(3 \times 10^{6}\right.$ cells/150-mm dish) followed by the addition of vehicle (ethanol, $0.1 \%$ ) or vehicle containing PF-3845 (10 $\mu \mathrm{M}$ final concentration). The culture medium was extracted using Clean-Up C18 solid-phase extraction columns (500 mg; United Chemical
Technologies, Bristol, PA). Briefly, the solid-phase extraction columns were conditioned with $3 \mathrm{ml}$ methanol followed by $3 \mathrm{ml}$ deionized water. The samples of culture medium $(25 \mathrm{ml})$ were then passed through the columns and washed, first using $3 \mathrm{ml}$ deionized water followed by $3 \mathrm{ml} 25 \%$ methanol and $3 \mathrm{ml} 60 \%$ methanol. The lipids eluting with $100 \%$ methanol were collected and evaporated under nitrogen, leaving approximately $500 \mu$ l. Fifty microliters was transferred to autosampler vials for reverse phase HPLC-UV analysis. Chromatographic separation was performed on a Grace Alltech C18 column $(100 \times 4.6 \mathrm{~mm}, 5 \mu \mathrm{m}$; Grace, Columbia, MD) using an HP1050 quaternary pump system. The mobile phases were as follows: A, water with $0.1 \%$ trifluoroacetic acid; and B, $100 \%$ methanol at a flow rate of $0.8 \mathrm{ml} / \mathrm{min}$. The following gradient was used: $0.0-0.5$ minutes, $50 \% \mathrm{~B} ; 0.5-5$ minutes, linear gradient to $100 \%$ B; and 5-15 minutes, holding at $100 \%$ B. Detection was by UV absorption at $205 \mathrm{~nm}$ using an HP1050 photodiode array detector.

Because the structure or structural class of the PF-3845-induced product (PIP) has not yet been determined and the isolated quantities were too low to permit accurate mass measurement, doses or dose rates for testing its biologic activities were expressed in AU units (milliabsorbance units $\times$ time in minutes) as determined by the ChemStation software (Agilent, Santa Clara, CA). Multiple batches of the isolated product were used for testing of biologic activity. The isolated product was stable after storage at $-20^{\circ} \mathrm{C}$ for more than 6 months.

Surgical Preparation for an Acute Renal Functional Mouse Model. Mice were anesthetized and prepared surgically for the acute renal function testing procedure as described previously (Zou et al., 2001; Li et al., 2005). Anesthesia was induced with ketamine (100 mg/ $\mathrm{kg}$, i.p.) and thiobutabarbital ( $75 \mathrm{mg} / \mathrm{kg}$, i.p.). The abdominal and neck areas were shaved, and the mice were placed on a thermostatically controlled warming table to maintain body temperature at $37^{\circ} \mathrm{C}$. A tracheotomy was performed by inserting a 2 -cm length of polyethylene PE-60 tubing to maintain a patent airway during surgery. After tracheotomy, a catheter prepared from heated and pulled plastic PE-10 tubing and filled with heparin sodium dissolved in saline (0.005 mg/ml, $50 \mathrm{IU} / \mathrm{ml}$; Fisher BioReagents, Pittsburgh, PA) was inserted into the left carotid artery and attached to a pressure transducer connected to a data acquisition system (Windaq; DATAQ Instruments, Akron, OH). The right jugular vein was catheterized with a catheter prepared from PE-10 tubing for the infusion of $0.9 \%$ saline containing $2 \%$ bovine serum albumin. The rate of intravenous infusion was $1 \mathrm{ml} / \mathrm{h}$ per $100 \mathrm{~g}$ body weight to maintain fluid volume and hematocrit concentration throughout the experiment. For the acute renal function experiments, an incision was performed following the midline of the abdominal area. The left ureter was ligated with a surgical suture and cut on the kidney side of the ligation to allow produced urine to exit freely into the abdominal cavity. The urethra was ligated in proximity to the bladder, and the latter was catheterized with a 2.5-cm cut length of PE-50 tubing for the collection of urine at timed intervals. The right kidney was elevated on a piece of sterilized gauze and a pulled PE-10 catheter was implanted to $2.5 \mathrm{~mm}$ in depth vertically from the dorsal surface into the outer medulla. The catheter was immobilized to the kidney capsule using a cyanoacrylate tissue adhesive (Vetbond; 3M Animal Care Products, St. Paul, MN). All mice received a 1-hour equilibration period, during which the animals received continuous vehicle infusions as follows: 1) intravenous infusion of filter-sterilized $0.9 \% \mathrm{NaCl}$ containing $2 \%$ albumin at a rate of $1 \mathrm{ml} / \mathrm{h}$ per $100 \mathrm{~g}$ body weight, 2) intramedullary infusion with PBS containing $205 \mathrm{mM} \mathrm{NaCl}, 40.5 \mathrm{mM} \mathrm{Na}_{2} \mathrm{HPO}_{4}$, and $9.5 \mathrm{mM}$

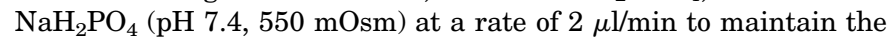
patency of interstitial infusion (Zhu et al., 2011). The urine excretion rate was measured gravimetrically, and the sodium and potassium concentrations in urine were measured using flame photometry. The urine excretion rate and the urinary sodium and potassium excretion rates ( $U_{\mathrm{Na}}$ and $\mathrm{U}_{\mathrm{K}}$, respectively) were factored per gram of kidney weight. At the end of each experiment, the animals were euthanized by exsanguination from the carotid artery catheter for collection of 
plasma. The placement of the intramedullary catheter in the outer medulla was routinely confirmed by postmortem kidney dissection after the experiments.

Experimental Protocols. After a 1-hour equilibration period and three sequential 10-minute pretreatment control sample collection periods, the lipid was infused intravenously or intramedullary over a 10-minute period, followed by one to five post-treatment control sample collections as indicated. The subjects received either an intravenous or intramedullary 10-minute infusion of the compound at doses ranging from $0.25 \mathrm{AU}$ to $8 \mathrm{AU}$. Sham mice received only vehicle infusions. The average between the 10-minute treatment and 10-minute washout periods was taken to represent the response to drug treatment. Some subjects received intraperitoneal and intravenous bolus injections of the compound after the vehicle pretreatment control periods as indicated for each experiment: group 1, continuous intravenous infusion of the PIP in increasing cumulative doses $(0.25$, $0.5,0.75,1,2,4$, and $8 \mathrm{AU})$ in C57BL/6J mice $(n=11)$; group 2 , continuous intramedullary infusion of $\operatorname{PIP}(0.25,0.5,0.75,1,2,4$, and $8 \mathrm{AU})$ in $\mathrm{C} 57 \mathrm{BL} / 6 \mathrm{~J}$ mice $(n=7)$; group 3, continuous intravenous vehicle infusion in C57BL/6J mice; group $4(n=7)$, continuous intramedullary vehicle infusion in C57BL/6J mice $(n=7)$; group 5 , intravenous infusion of PIP (1 AU/10 min), followed by three 10 -minute post-treatment recovery periods $(n=4)$; group 6 , intravenous bolus injection of $5 \mathrm{AU}$ in $20 \mu \mathrm{l}$ vehicle, followed by a 50 -minute recovery period $(n=4)$; group 7 , intraperitoneal bolus injection of $5 \mathrm{AU}$ in $20 \mu \mathrm{l}$ vehicle, followed by a 50 -minute recovery period $(n=4)$; group 8, intravenous infusion of PIP $(0.25,0.5,1,2,4$, and $8 \mathrm{AU})$ in FAAH WT and FAAH KO mice ( $n=6$ and $n=7)$; and group 9, sham, intravenous, or intraperitoneal bolus injections with $20 \mu$ l vehicle alone, followed by a 50 -minute recovery period $(n=8)$.

Glomerular Filtration Rate. The glomerular filtration rate (GFR) was determined by urinary clearance of fluorescein isothiocyanate (FITC)-labeled sinistrin ( $3 \mu \mathrm{g} / \mathrm{min}$ per kilogram in $0.9 \%$ saline/ $2 \%$ bovine serum albumin, FITC-sinistrin; Fresenius, Kabi, Austria). The compound was infused intravenously at a constant rate starting during the 1-hour acclimation period before the experiment. Arterial blood was sampled at the beginning of the vehicle infusion and at the end of the experiment. The blood samples were centrifuged for 2 minutes at $1000 \mathrm{~g}$ in a microcentrifuge. Urine samples were collected from the bladder catheter every 10 minutes. FITC fluorescence was determined with an automated fluorescence microplate reader (FLx800) and Gen5 data analysis software (both from BioTek Instruments, Winooski, VT).

Medullary Blood Flow. For the measurement of medullary blood flow (MBF), a laser Doppler flow probe (OxyFlo Pro, MNP 100XP; Oxford Optronix, Oxford, UK) was stabilized on the ventromediolateral surface of the right kidney to measure the MBF continuously (as previously reported in Ahmad et al., 2017, 2018). The recording of the blood flow was measured by using a dual-channel laser Doppler flowmeter (Transonic Systems Inc., Ithaca, NY).

Statistical Analysis. Data are presented as the mean \pm S.E.M. For comparison between multiple groups of data, one- or two-way analysis of variance was applied using the Tukey, Dunnett, or Fisher least significant difference (where appropriate) post hoc test when significant differences were found. A $P$ value $\leq 0.05$ was considered statistically significant.

\section{Results}

Characterization of Cultured MMICs. The MMIC cell population established in our study stained positively for tenascin C (Fig. 1A), whereas cells stained only with the HRPconjugated secondary antibody were negative (Fig. 1B). To confirm the report of $\mathrm{He}$ et al. (2013) that tenascin $\mathrm{C}$ is a selective marker for medullary interstitial cells, sections of mouse kidney were also tested. Positive immunostaining by the tenascin $\mathrm{C}$ antibody was restricted to the inner medulla/papilla (Fig. 1C); the outer medulla (Fig. 1D) and cortical region (not shown) were negative. This immunostaining in the inner medulla was associated with the ladder-like structures of the medullary interstitial cells, which exhibited oblong nuclei on an axis perpendicular to the tubules of the inner medulla. The cultured MMICs also exhibited intense staining for COX-2 (Fig. 1E) and they stained positively for the presence of FAAH (Fig. 1F). In addition, the cultured MMICs also exhibited abundant cytoplasmic granules visible by phase-contrast light microscopy. Positive histochemical staining of cytoplasmic vesicles with Oil Red O and Sudan Black B (Fig. 1, G and H) is consistent with the presence of neutral lipid-containing granules in the MMICs.

The apparent size and number of these vesicles varied over time, being most prominent after fresh medium changes. They also showed selective responses to drug treatments. Treatment with the FAAH inhibitor, PF-3845, increased the number and size of these Sudan Black B-stained granules, whereas no changes were evident after treatment with the COX inhibitor, celecoxib. Cotreatment with PF-3845 and celecoxib appeared to cause further proliferation of these lipid granules (Fig. 2). The onset of the PF-3845 effect on the Sudan Black B staining pattern was evident within 2-4 hours after the start of treatment and was dose dependent from 1 to $20 \mu \mathrm{M}$ PF-3845 (Fig. 2). These PF-3845-induced lipid granules typically showed the greatest accumulation in the perinuclear region.

Analysis of MMICs by Transmission Electron Microscopy. Ultrastructural analysis of the MMICs by transmission electron microscopy revealed large prominent nuclei, abundant mitochondria, and dilated rough endoplasmic reticulum in their cytoplasm (Fig. 3). Stacks of Golgi apparatus could be found. The cytoplasm was rich in granular and vesicular elements, consistent with lipid droplets, vacuoles, lysosomes, and other structures. Multilamellar bodies with their characteristic whorled appearance were visible in some images of control MMICs (Fig. 3B, arrows). Microvilli were apparent on the plasma membrane. Similar analyses of PF-3845-treated MMICs revealed similar general characteristics as described above, with two notable exceptions. The PF-3845-treated MMICs exhibited pronounced nucleoplasmic invaginations (Fig. 3, C and D) and more numerous and larger multilamellar bodies (Fig. 3, C and D, arrows).

Identification of an Apparent Novel PIP of MMICs by Reverse Phase HPLC. During experiments to assess the effects of PF-3845 treatment on the MMIC secretion of prostaglandin $\mathrm{E}_{2}$, prostamide $\mathrm{E}_{2}$, and anandamide, a difference was noted in the chromatographic profile of lipid extracts from the medium of PF-3845-treated versus control MMICs (Fig. 4A). A peak at 13.8 minutes was increased by PF-3845 treatment compared with the control. In contrast, treatment with a COX inhibitor had no effect, whereas cotreatment of cells with PF-3845 and a COX inhibitor reduced the area of this peak. Quantitation of the effect based on area under the peak is shown in Fig. 4B. Further analyses showed that the product was present in complete growth medium (DMEM supplemented with fetal calf serum) and that it was increased in the medium from MMIC cultures incubated for 24 hours, suggesting that MMICs can produce and secrete this product. The area of the peak was further increased by treatment with PF-3845. Dose-response analysis indicated a maximal 2.6-fold increase at $10 \mu \mathrm{M}$ and a smaller but significant 0.4 -fold increase at $20 \mu \mathrm{M}$ (Fig. 4C). 

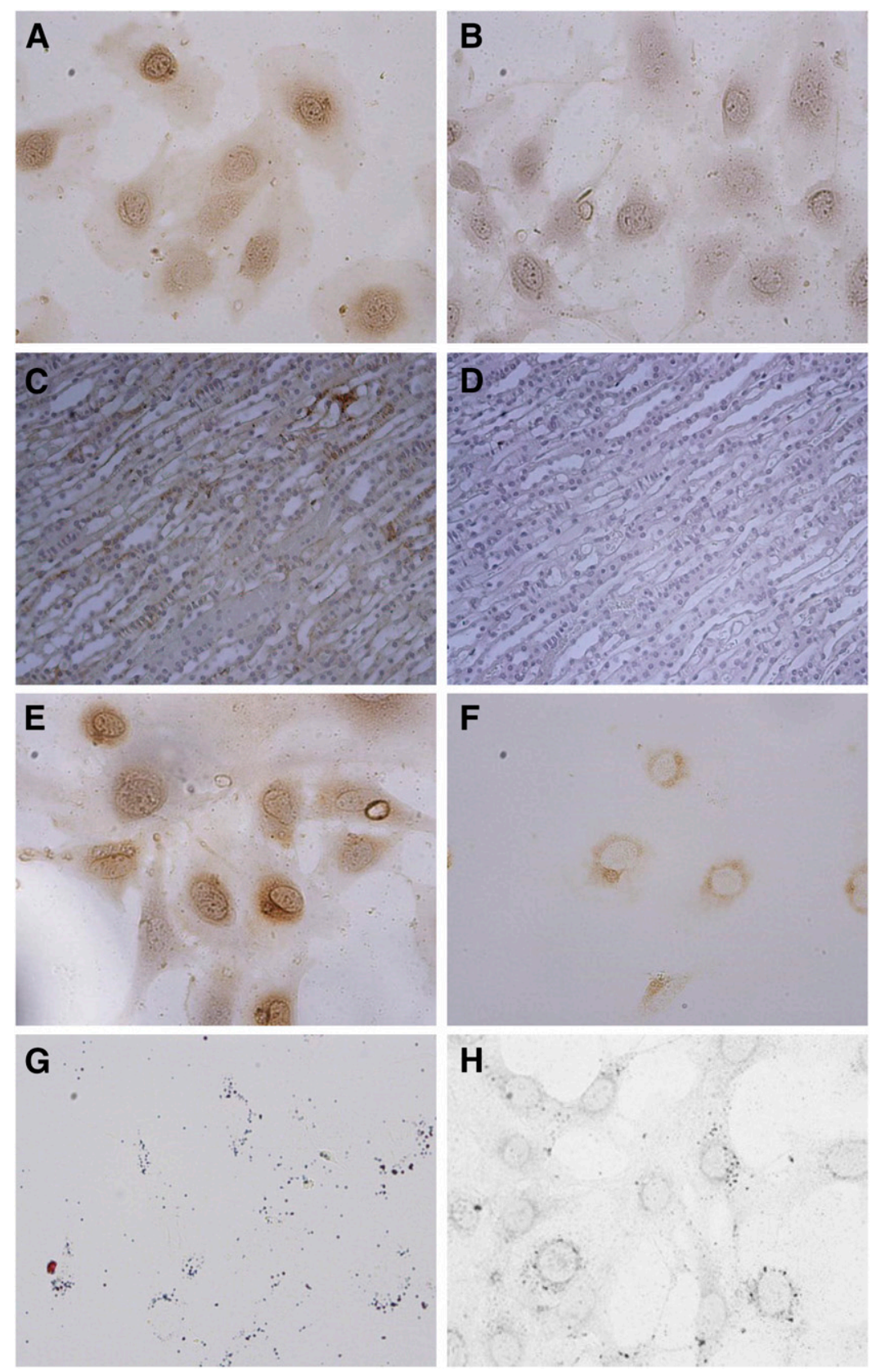

Fig. 1. Immunohistochemical and histochemical analysis of cultured MMICs and kidney tissue sections. Cultured MMICs or kidney tissue sections were subjected to immunohistochemical or histochemical analyses as described. Representative images of analyses performed in triplicate are shown. (A) MMICs stained with tenascin C primary antibody. (B) Negative control MMICs stained only with HRP-conjugated secondary antibody. (C) Section of the inner medullary region of mouse kidney stained for tenascin C antibody. (D) Section of the outer medullary region stained for tenascin C. (E) COX-2-stained MMICs. (F) FAAH-stained MMICs. (G) Oil Red O-stained MMICs. (H) Sudan Black B-stained MMICs. Original magnification, $200 \times$.
Effects of the PIP Fraction in an Acute Renal Function Model Using C57BL/6J Mice. To characterize the effects of the PIP fraction on acute renal function, the product was isolated from the culture medium of MMICs exposed to $10 \mu \mathrm{M}$ PF-3845 for 24 hours and tested for its effects on blood pressure and urinary parameters (Fig. 5). Figure 5A presents the effects of the PIP fraction given by intravenous infusions on MAP, urine excretion rate, $U_{\mathrm{Na}}$, and $\mathrm{U}_{\mathrm{K}}$ in $\mathrm{C} 57 \mathrm{BL} / 6 \mathrm{~J}$ mice. For controls, sham-treated mice were included that received only vehicle for the duration of the experimental period but were otherwise identically treated. Neither MAP nor the urine excretion rate was significantly affected over time in the sham control mice. The effects of the PIP fraction given intravenously in 10-minute infusions at doses ranging from 0.25 to 8 total AU are shown in Fig. 5A. The average MAP for the pretreatment control phases, C1 and
C2, was 115 and $112 \mathrm{mmHg}$, respectively. Although PIP did not significantly change MAP at dose rates of $0.25-4$ AU/10 min, there was a decreasing trend in MAP. By the $8 \mathrm{AU}$ dose rate, this decrease was significant $(96 \mathrm{~mm} \mathrm{Hg}$; $P<0.05$ ), and MAP remained low during the two following post-treatment phases (96 and $91 \mathrm{~mm} \mathrm{Hg}$ for P1 and P2, respectively; $P<0.0001)$. This drop in blood pressure was also significant compared with the sham control group. Intravenous infusion of increasing doses of PIP to the same mice also enhanced the rates of urine formation and sodium excretion, but the changes were not dose dependent and the dose-response curves exhibited an inverted U-shape. For example, the urine excretion rate increased from $7 \mu \mathrm{l} / \mathrm{min}$ per gram of kidney weight in the $\mathrm{C} 1$ and $\mathrm{C} 2$ control groups to 20,16 , and $18 \mu \mathrm{l} / \mathrm{min}$ per gram of kidney weight, respectively, for the $0.25,0.5$, and $0.75 \mathrm{AU}$ dose groups, only to 

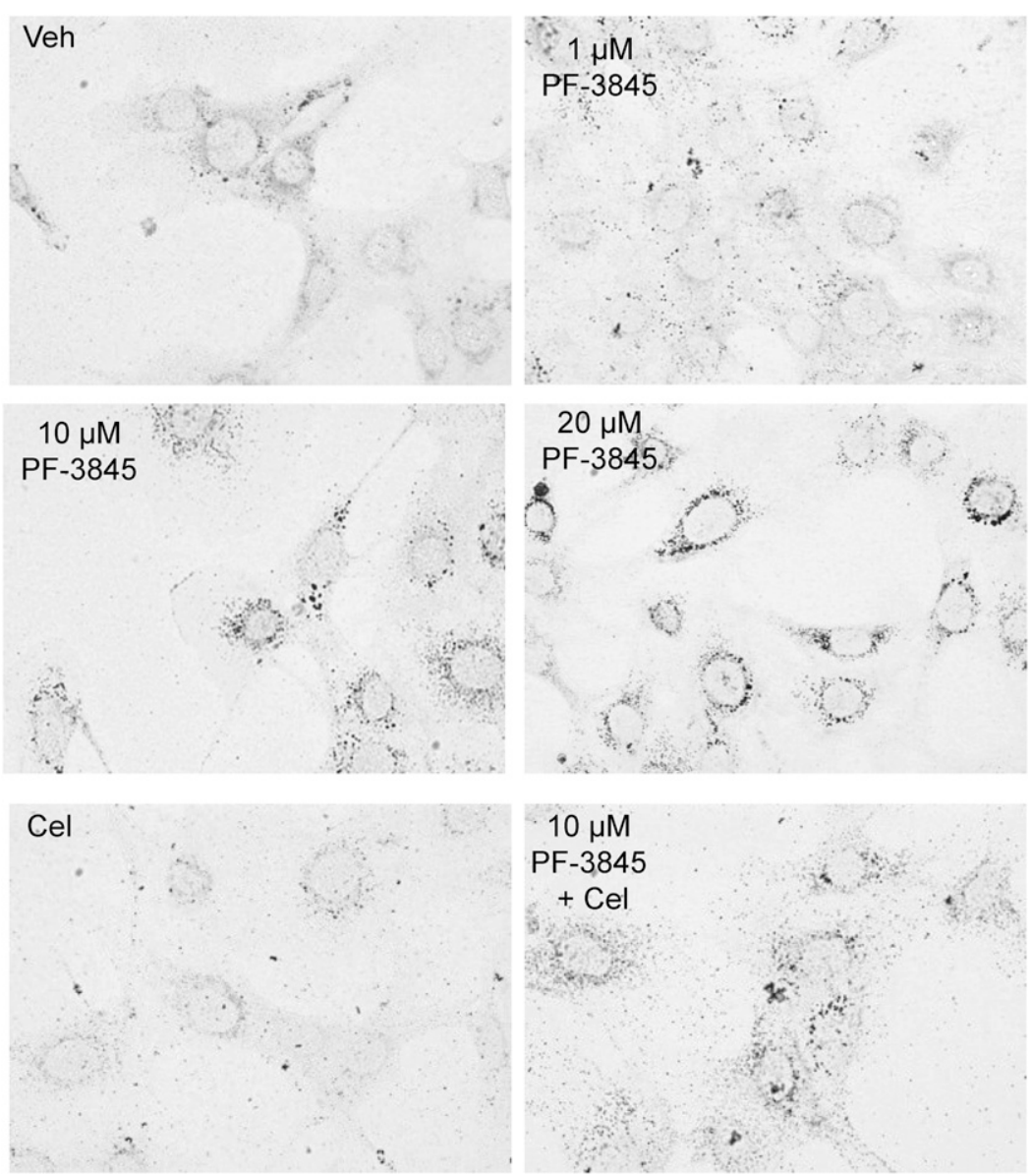

Fig. 2. The effect of PF-3845 in the presence or absence of a COX-2 inhibitor on lipid staining in cultured MMICs. MMICs cultured in the presence of vehicle ( $0.1 \%$ ethanol), $\mathrm{PF}-3845(1,10$, or $20 \mu \mathrm{M})$, celecoxib $(10 \mu \mathrm{M})$, or PF-3845 plus celecoxib for 24 hours were stained with Sudan Black $\mathrm{B}$ as described in the Materials and Methods. Representative images of analyses performed in triplicate are shown. Cel, celecoxib; Veh, vehicle. Original magnification, $200 \times$. return back toward the control level with the higher PIP doses and during the P1 and P2 post-treatment control phases. A similar absence of dose dependence and the presence of an inverted U-shaped curve were apparent for the effect of intravenous PIP on sodium excretion. Potassium excretion was not significantly altered during the time course of the experiment. The same effects were observed using PIP isolates from multiple batches of PF-3845-treated MMIC cell medium on at least five different dates (data not shown).
When the PIP fraction was infused directly into the renal medullary interstitium, MAP was also reduced (Fig. 5B). A similar pattern to the intravenous response was observed with a downward trend in MAP apparent at the low end of the dose range, which reached significance as the dose increased. The MAP of the $\mathrm{C} 1$ and $\mathrm{C} 2$ groups was 119 and $117 \mathrm{mmHg}$, which decreased to 103,99 , and 93 after intramedullary infusion of 2 , 4 , and 8 AU/10 $\mathrm{min}$ of the PIP fraction, respectively. The average arterial pressures remained low in the P1 and P2 post-treatment groups $(P<0.01)$. Urine excretion increased
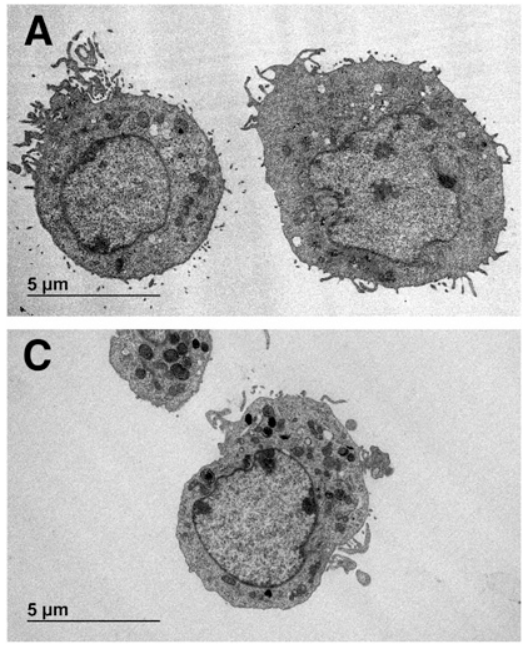
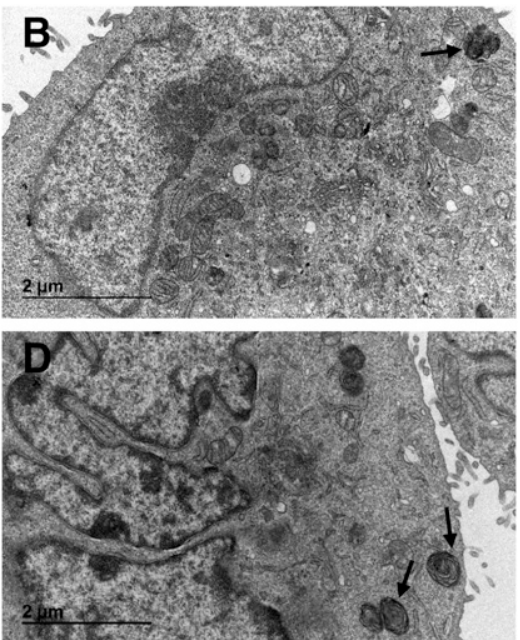

Fig. 3. Transmission electron micrographs of vehicle- and PF-3845-treated MMICs. (A-D) MMICs treated with vehicle (0.1\% ethanol) for 24 hours (A and B) or with $10 \mu \mathrm{M}$ PF-3845 for 24 hours (C and D). Low (A and C) and high (B and D) magnification images are shown for each. Osmiophilic lipid granules are seen in the vehicle- and PF-3845-treated MMIC images, including multilamellar bodies (arrows). Cytoplasmic invaginations were also a frequent feature of the PF-3845-treated cells. 

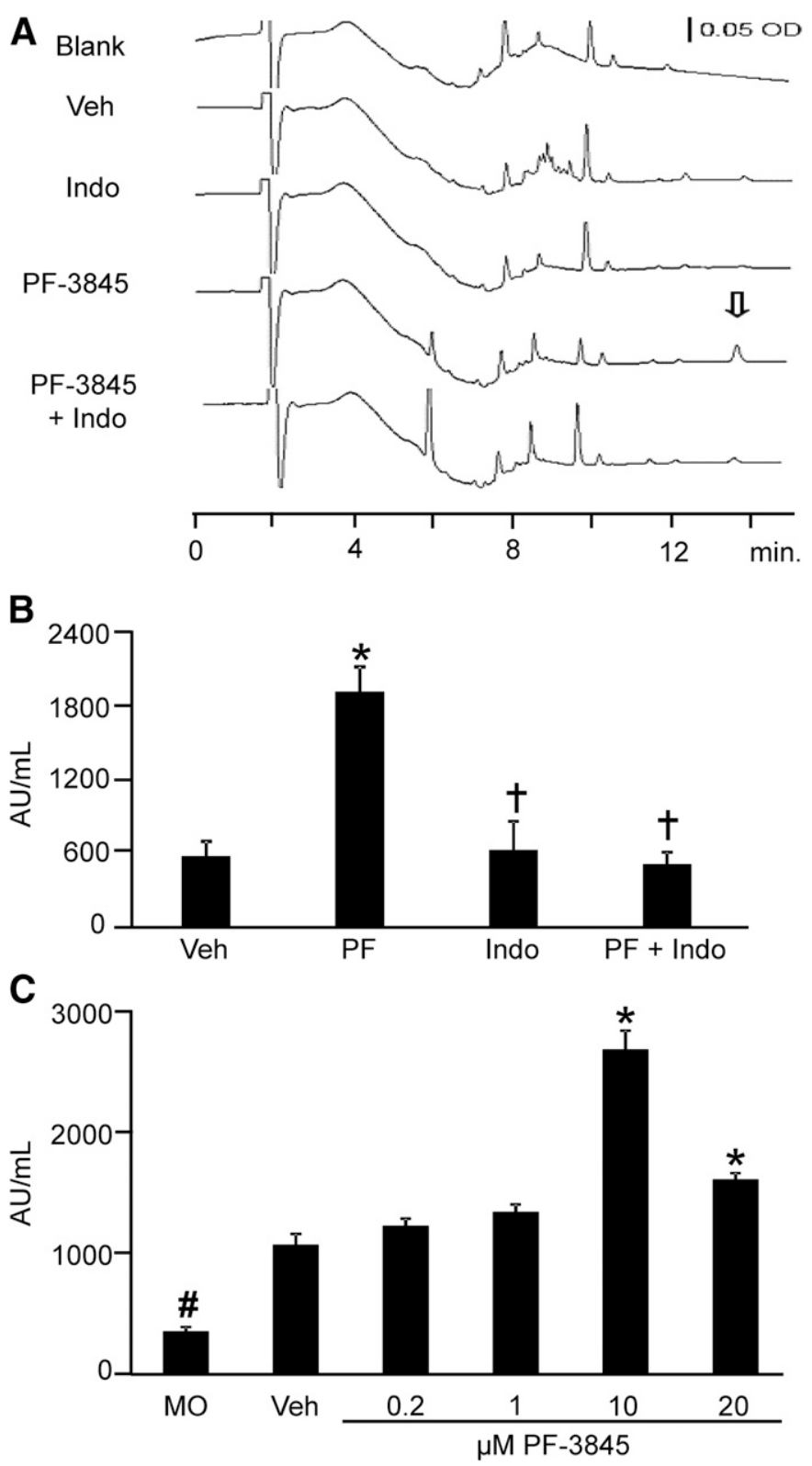

Fig. 4. Identification of a lipophilic product in the culture medium of MMICs that shows responsiveness to treatment with PF-3845. (A) Representative chromatograms of negative control (blank methanol) and lipid extracts from MMICs treated with vehicle, indomethacin $(10 \mu \mathrm{M})$, PF-3845 $(10 \mu \mathrm{M})$, or the combination (indomethacin and PF-3845) with the UV detector set at $205 \mathrm{~nm}$. The arrow indicates a PIP eluting at approximately 13.8 minutes (designated PIP). (B) Relative quantitative data of the treatment effects on PIP. Bars represent the mean \pm S.E. of the mean of the area under the PIP peak normalized for the volume of culture medium extracted. ${ }^{*} P<0.05$ indicates a significant difference vs. the vehicle group (one-way ANOVA with Tukey post hoc test); ${ }^{\dagger} P<0.05$ indicates a significant difference vs. the PF-3845 treatment group $(n=5$ independent samples). (C) Concentration response of the PF-3845 effect on PIP. Treatments at the indicated concentrations were for 24 hours. ${ }^{*} P<0.05$ indicates a significant difference vs. vehicle (one-way ANOVA with the Dunnett post hoc test; $n=5$ per treatment group); ${ }^{\#} P<0.05$ indicates a significant difference between the medium-only and vehicle control groups. ANOVA, analysis of variance; PF, PF-3845; Indo, indomethacin; MO, medium only; Veh, vehicle.

from 15 to $14 \mu \mathrm{l} / \mathrm{min}$ per gram of kidney weight for $\mathrm{C} 1$ and C2 to 20,26 , and $29(P<0.0001)$ for the $0.25,0.5$, and 0.75 AU intramedullary doses, respectively. At the 1 AU dose or higher, the magnitude of the effect decreased, returning to the baseline level by $2 \mathrm{AU}$ and remaining at baseline after the two highest doses and in the P1 and P2 post-treatment groups. This difference in urine excretion in the PIP fraction-treated mice was also significant versus the corresponding points for the sham control mice $(P<0.0001)$. Whereas the sodium excretion rate also showed a similar stimulation at the low but not high doses, the potassium excretion rate was not significantly affected.

Figure 6 presents the results for a single intravenous infusion of the PIP fraction (1 AU/10 $\mathrm{min}$ ) on acute renal function, including GFR and MBF. Although MAP was not changed during the actual drug infusion period, MAP decreased significantly on the order of $10 \mathrm{~mm} \mathrm{Hg}$ during each of the three postinfusion periods (Fig. 6A; $P<0.05$ ). The urine excretion rate was increased beginning within the first 10-minute post-treatment time period and remaining elevated thereafter (Fig. 6B; $P<0.001$ ). The baseline GFR values for $\mathrm{C} 1, \mathrm{C} 2$, and $\mathrm{C} 3$ were $1.8,1.5$, and $1.7 \mathrm{ml} / \mathrm{min}$, respectively, whereas the single $1 \mathrm{AU}$ infusion stimulated GFR to $3.6 \mathrm{ml} /$ min (Fig. 6C), remaining elevated for the immediate term thereafter $(P<0.0014)$. GFR began dropping back to baseline levels after 40 minutes. Treatment with the PIP fraction also significantly elevated MBF (Fig. 6D; $P<0.01$ ) but this stimulation was brief, returning to the control level by the P1 post-treatment period.

Effects of Intravenous or Intraperitoneal Bolus Administrations of the PIP Fraction on MAP, Urine Excretion Rate, and MBF. The effects of the PIP fraction were also evaluated after bolus administrations given either intravenously or intraperitoneally (Fig. 7). For the intravenous bolus group, the baseline MAP readings were $110 \mathrm{~mm} \mathrm{Hg}$ for each of the three control groups (vehicle, C1, and C2; Fig. 7A) and intravenous bolus administration of $5 \mathrm{AU}$ did not change blood pressure after the dose, even after administering a second 5 AU dose. Whereas MAP showed no response to intraperitoneal injection of vehicle alone (ethanol) and remained at 102 and $102 \mathrm{~mm} \mathrm{Hg}$ for the $\mathrm{C} 1$ and $\mathrm{C} 2$ periods with respect to the starting MAP of $107 \mathrm{~mm} \mathrm{Hg}$, the blood pressure was lowered by a intraperitoneal 5 AU bolus injection of the PIP fraction to 90 , then to $83 \mathrm{~mm} \mathrm{Hg}$, and remained there for at least 50 minutes after the dose. Subsequent administration of a second intraperitoneal 5 AU bolus dropped the pressure initially to 79 , then 72 and $71 \mathrm{~mm} \mathrm{Hg}$.

The urine excretion rate data for intravenous versus intraperitoneal 5 AU bolus dosing showed a striking contrast to the MAP results (Fig. 7B). Whereas the baseline urine excretion rates for the vehicle, $\mathrm{C} 1$, and $\mathrm{C} 2$ control groups of the intravenous bolus treatment group were 11,12 , and $11 \mu \mathrm{l} /$ min per gram of kidney weight, respectively (Fig. 7B), the urine excretion rate increased to $19 \mu \mathrm{l} / \mathrm{min}$ per gram of kidney weight $(P<0.005)$ after the intravenous 5 AU bolus and remained elevated during the subsequent post-treatment periods (26-28 $\mu \mathrm{l} / \mathrm{min}$ per gram of kidney weight, P1-P3). A second bolus of the same intravenous $5 \mathrm{AU}$ dose given 50 minutes later did not further increase the urine excretion rate, but the urine excretion rate remained elevated. In contrast, the urine excretion rate was unaffected by either a first or second intraperitoneal 5 AU bolus injection. The urine excretion rate in the sham group was not significantly affected at any time of the experiment. To test the effects of the intravenous and intraperitoneal PIP fraction on medullary hemodynamics, MBF was also measured. MBF 

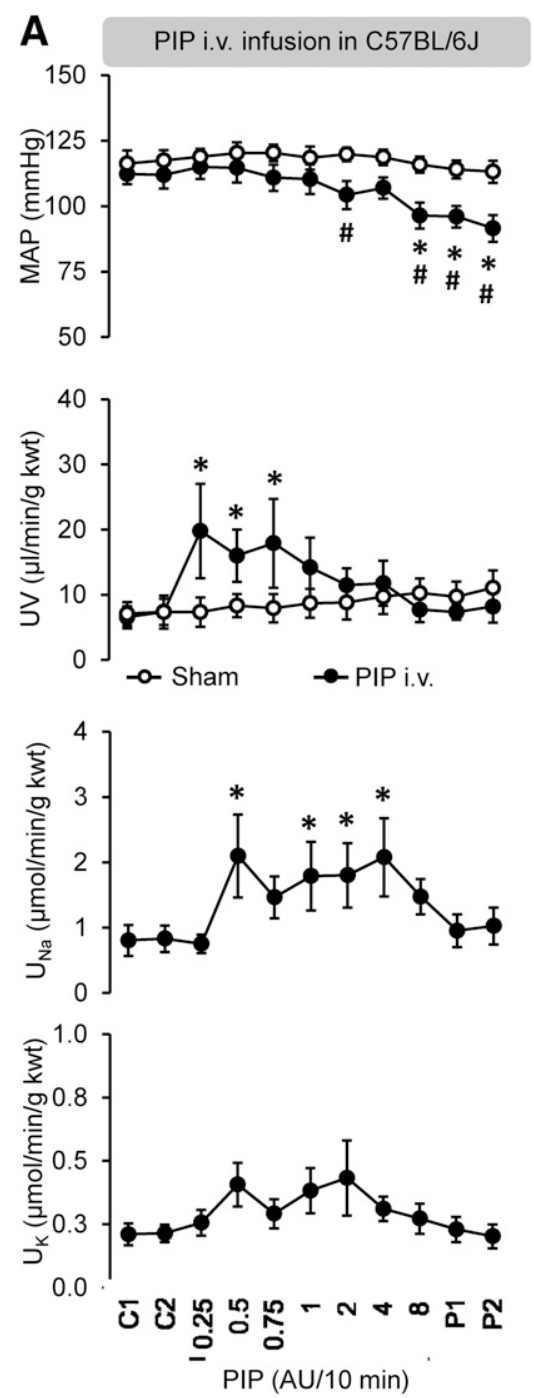
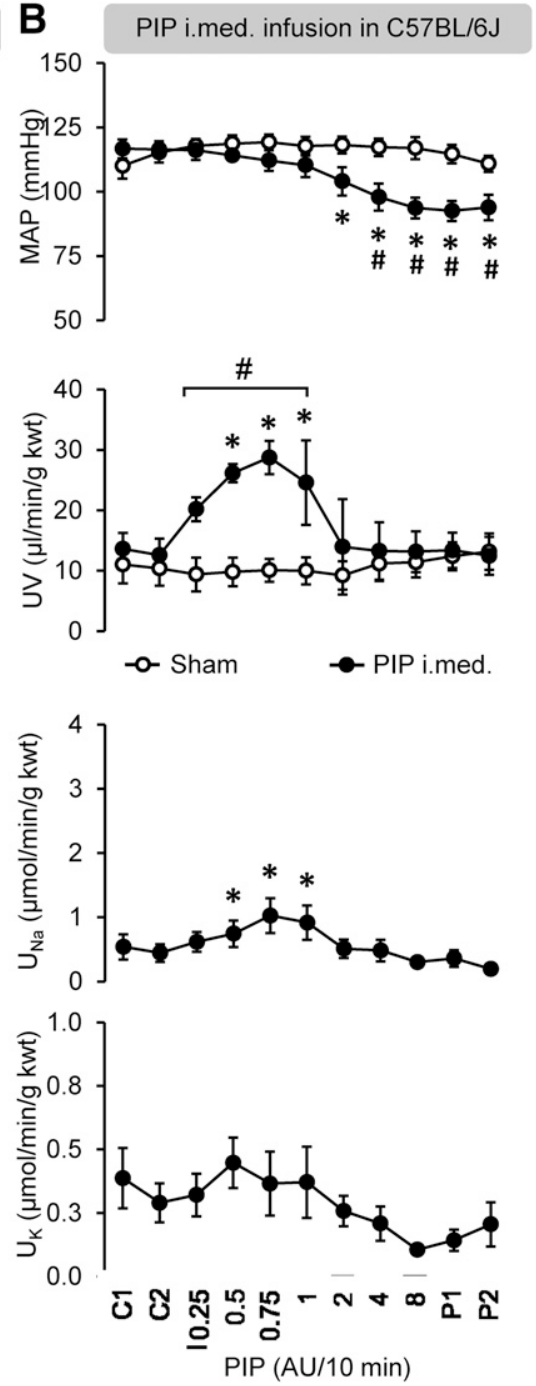

Fig. 5. The effects of intravenous (A) and intramedullary (B) infusion of the PIP fraction on MAP, urine excretion rate, $\mathrm{U}_{\mathrm{Na}}$, and $\mathrm{U}_{\mathrm{K}} . \mathrm{C} 1$ and $\mathrm{C} 2$ indicate the pretreatment control phases; P1 and P2 indicate the two 10-minute posttreatment control periods. Animals received the PIP fraction at the indicated doses by infusion over a single 10-minute period, followed by a 10-minute infusion of vehicle alone. Each data point represents the mean of the 20 -minute period. Sham mice received only vehicle the entire experimental period. ${ }^{*} P<0.05$ indicates a significant difference vs. the $\mathrm{C} 1$ group (one-way ANOVA with the Dunnett and Fisher post hoc tests); ${ }^{\#} P<0.05$ indicates a significant difference between the PIP and sham groups (one-way ANOVA with the Tukey post hoc test; $n=7$ for sham, $n=7$ for intramedullary infusion, and $n=11$ for intravenous infusion). ANOVA, analysis of variance; kwt, kidney weight; UV, urine excretion rate. was differentially stimulated when the PIP fraction bolus was administered intraperitoneally compared with intravenously (Fig. 7C).

Characterization of the PF-3845-Induced MMIC Product in an Acute Renal Function Animal Model in FAAH WT and FAAH KO Mice. The responses of MAP and urinary excretion parameters to the PIP fraction were also studied in FAAH KO mice and their homozygous normal littermates (FAAH WT) (Fig. 8). The baseline MAP of FAAH $\mathrm{KO}$ mice after the 1-hour acclimatization period was 120 and $122 \mathrm{~mm} \mathrm{Hg}$ for the $\mathrm{C} 1$ and $\mathrm{C} 2$ pretreatment control periods, respectively. MAP was lowered significantly after the $8 \mathrm{AU}$ dose and during the following $\mathrm{P} 1$ and $\mathrm{P} 2$ post-treatment periods (Fig. 8A). There were no effects of the PIP fraction on the urine excretion rate, $\mathrm{U}_{\mathrm{Na}}$, or $\mathrm{U}_{\mathrm{K}}$ in the FAAH $\mathrm{KO}$ mice (Fig. 8, B-D, respectively).

\section{Discussion}

This study characterized the diuretic, natriuretic, and vasodepressor activity of a substance produced by mouse renomedullary interstitial cells that showed responsiveness to the FAAH inhibitor, PF-3845. Testing of the HPLC-purified product on acute renal functions in anesthetized normotensive mice showed that the product possessed blood pressure-lowering and diuretic and natriuretic activities, but that the profile of its effects differed depending on the route of administration. Its contrasting biologic effects after intraperitoneal administration suggest a role for liver metabolism in determining its biologic actions. Although the structure of the substance responsible for the biologic activities in the PIP fraction has not yet been identified, the finding that its concentration in MMIC culture medium increased by PF-3845 treatment and that its diuretic and natriuretic activities were attenuated in FAAH KO (Fig. 8, $\mathrm{B}$ and $\mathrm{C}$ ) mice suggest a relationship to anandamide or other fatty acid ethanolamides or amides. These data support the existence of a substance secreted by renomedullary interstitial cells that has the capacity to lower blood pressure by either renal or extrarenal mechanisms.

The product characterized in this study was detected in culture medium of MMICs based on its UV absorption at $205 \mathrm{~nm}$ and its enhancement by treatment with PF-3845. Further experiments showed that it was also present in complete culture medium but was further enhanced by incubation with MMICs and by PF-3845 treatment, supporting that it is a product of MMICs. Based on its unique retention time of 13.8 minutes, the substance is distinct from anandamide $(7.7$ minutes $)$ and prostaglandin $\mathrm{E}_{2}$ 
A

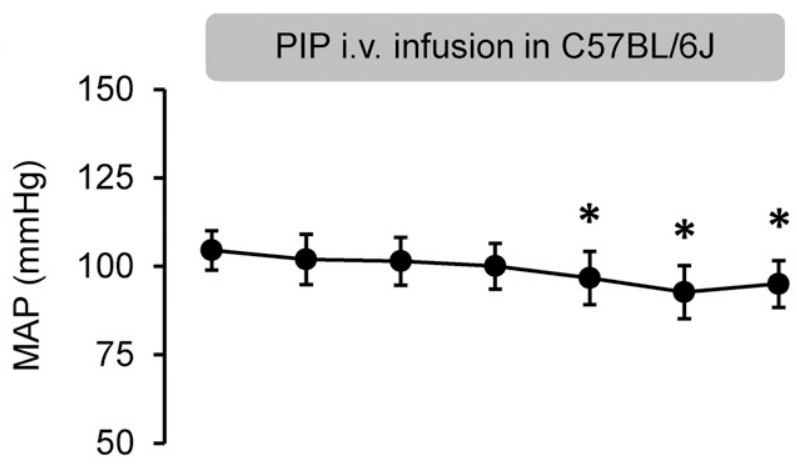

B

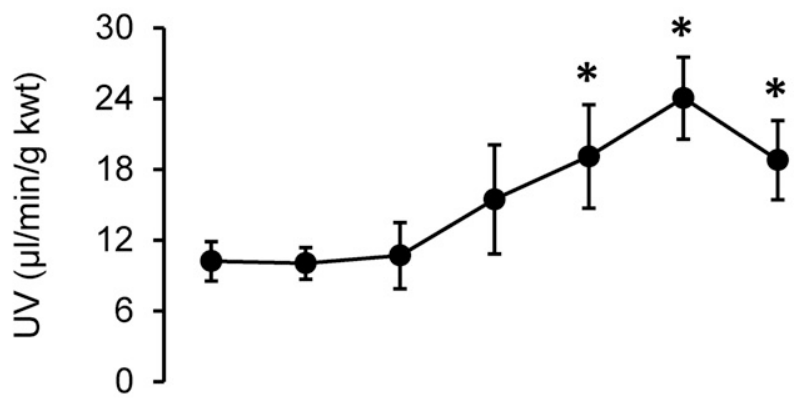

C

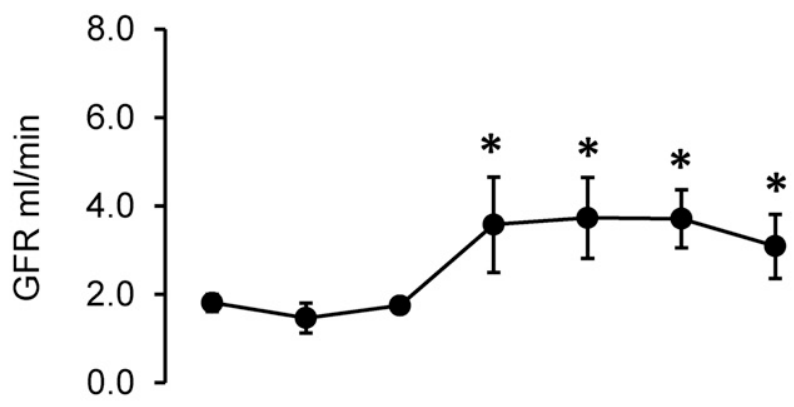

D

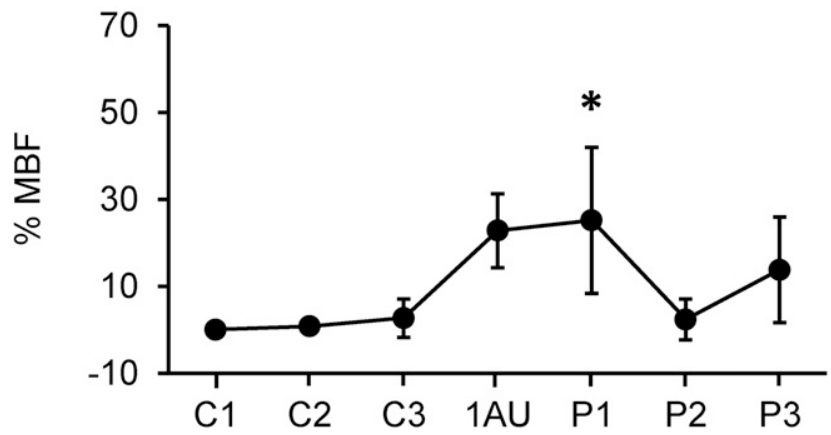

Fig. 6. The effects of a single intravenous infusion of the PIP fraction on (A) mean arterial pressure, (B) urine excretion rate, and (C) medullary blood flow. C1-C3 indicate the pretreatment control phases, during which the animals received intravenous vehicle only ( $2 \%$ bovine serum albumin in $0.9 \%$ saline). Animals then received a single intravenous infusion of 1 AU PIP fraction over a 10-minute period followed by three 10-minute post-treatment control infusion periods (P1-P3) with the vehicle alone. Each data point represents the mean of a 10 -minute period. $* P<0.05$ indicates a significant difference vs. the $\mathrm{C} 1$ group (one-way analysis of variance with Dunnett post hoc tests; $n=4$ ). kwt, kidney weight; UV, urine excretion rate.

(5.8 minutes). The decreased level of the product in indomethacin-treated cultures (Fig. 4, A and B) suggests that prostaglandin synthesis or metabolism by COX may be necessary for its formation or release.

The PIP fraction exhibited the capacity to stimulate diuresis and natriuresis, glomerular filtration, and $\mathrm{MBF}$ and to drop
A

PIP i.p. and i.v. bolus in C57BL/6J
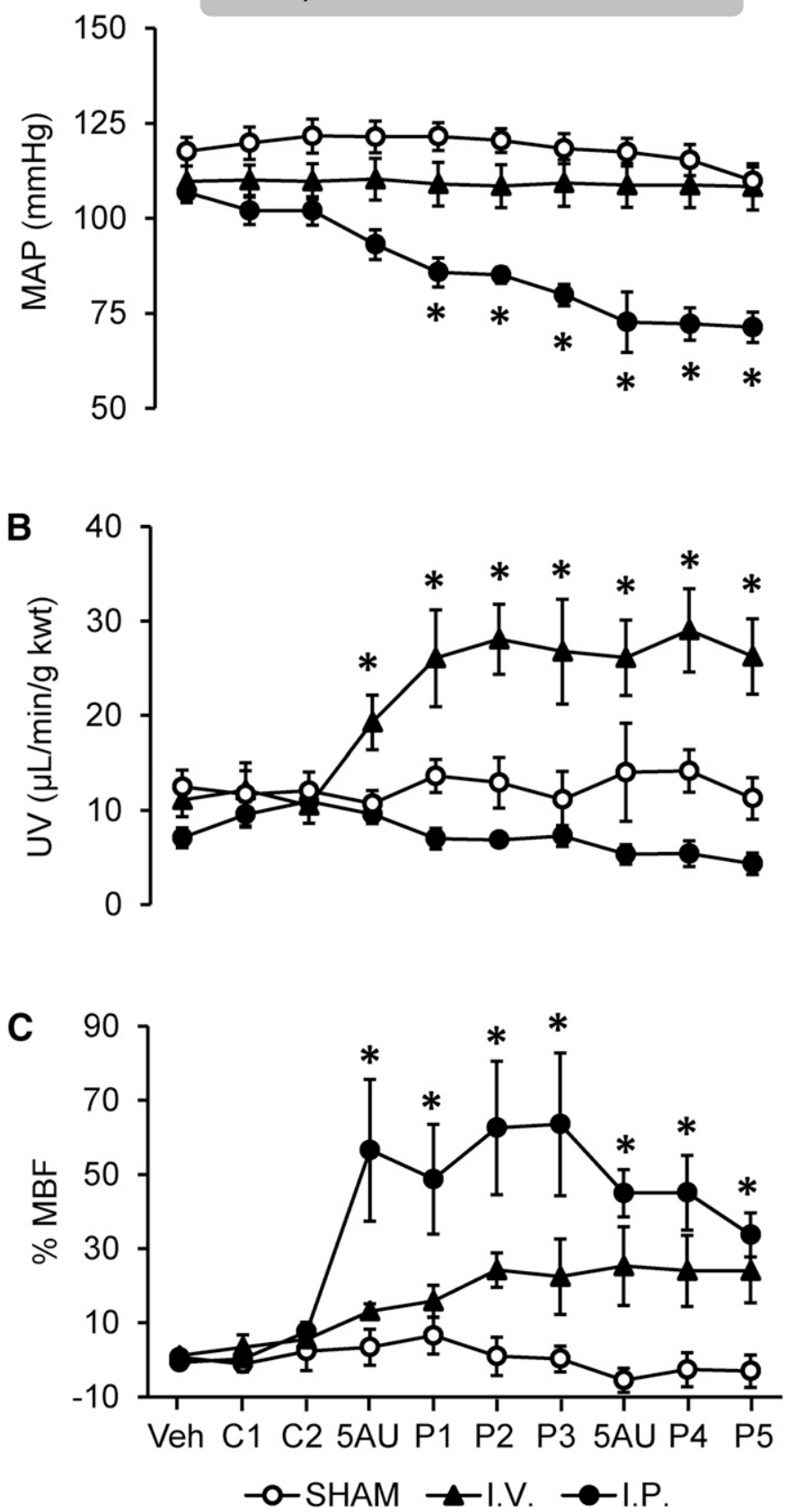

Fig. 7. The effects of intravenous and intraperitoneal bolus administrations of the PIP fraction on (A) mean arterial pressure, (B) urine excretion rate, (C) glomerular filtration rate, and (D) medullary blood flow. Sham animals (open circles) received only continuous infusions of vehicle throughout the entire experimental period. Animals received either an intravenous (closed triangles) or intraperitoneal (closed circles) bolus dose of vehicle followed by injections of 5 AU PIP fraction. Boluses were delivered over a period of 8-15 seconds. The vehicles for intravenous and intraperitoneal administrations were $2 \%$ bovine serum albumin/ $0.9 \%$ saline and ethanol, respectively. $\mathrm{C} 1$ and $\mathrm{C} 2$ are postvehicle control phases, and P1-P5 are 10-minute post-treatment control periods. ${ }^{*} P<0.05$ indicates a significant difference compared with the $\mathrm{C} 1$ group (one-way analysis of variance with the Dunnett post hoc test; $n=4$ for intraperitoneal administration, $n=4$ for intravenous administration, and $n=8$ for sham). kwt, kidney weight; UV, urine excretion rate; Veh, vehicle.

blood pressure in the surgically instrumented mouse model used in this study. However, there were notable differences depending on the route of administration. The diuretic activity 
A

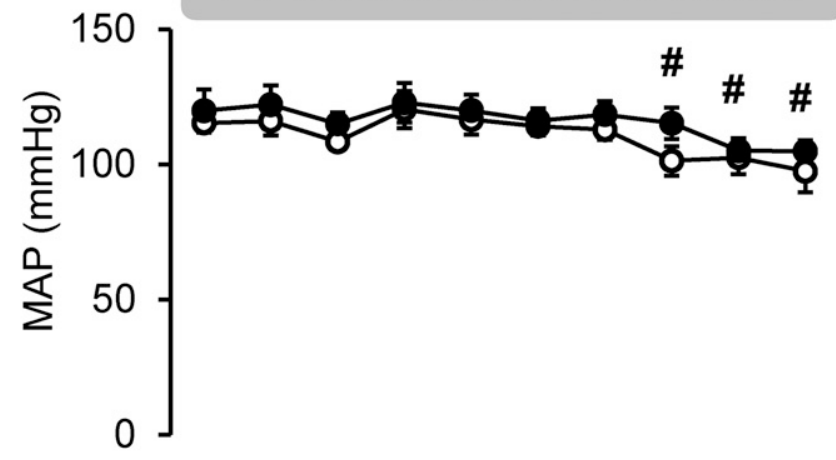

B

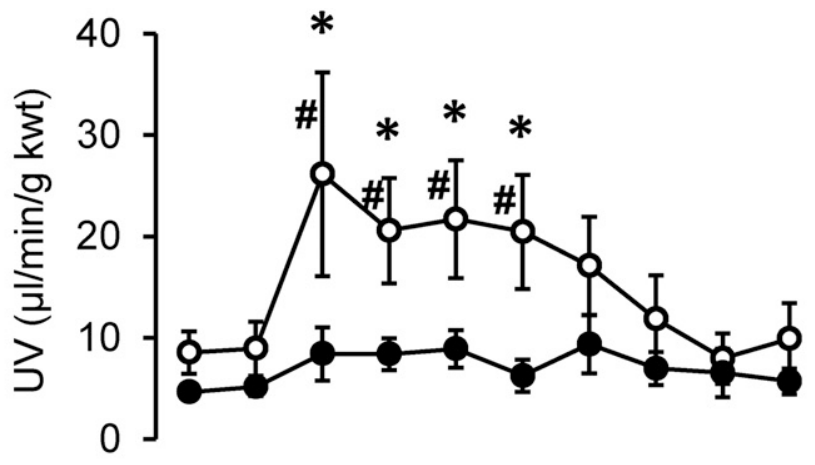

$c$
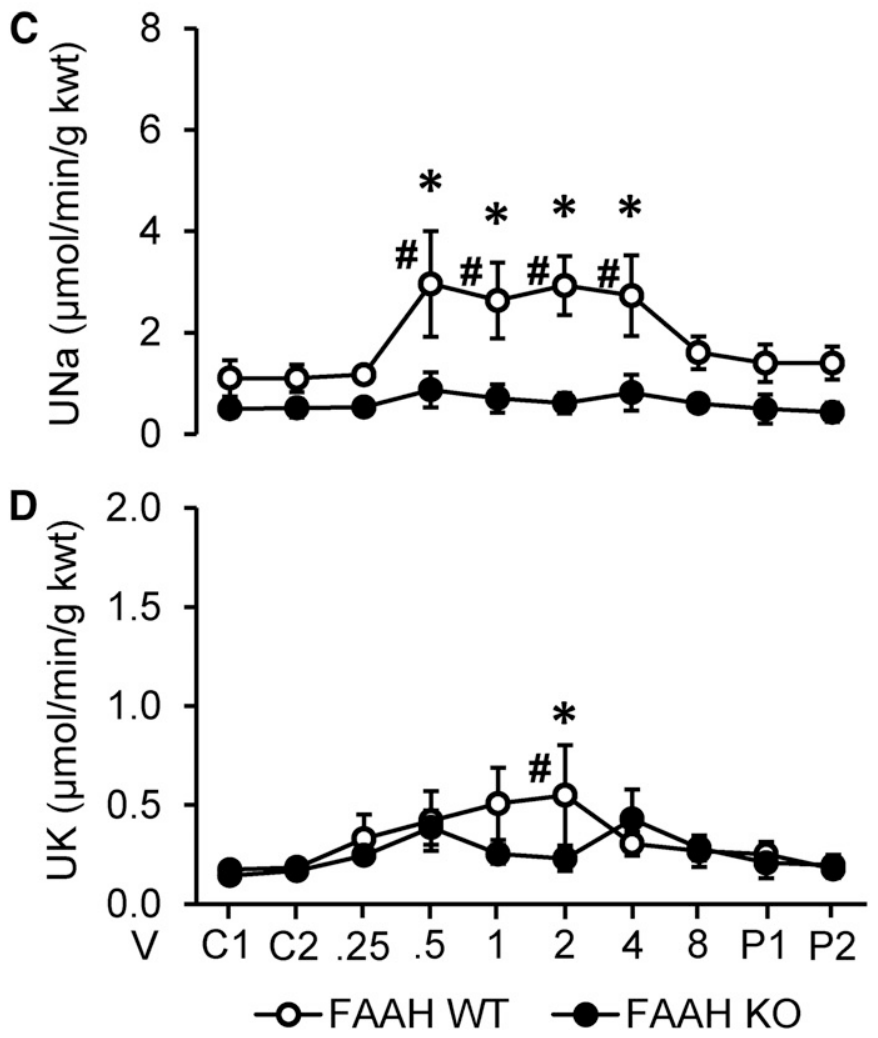

Fig. 8. Comparison of the effects of intravenous infusion of the PIP fraction on (A) mean arterial pressure, $(B)$ urine excretion rate, $(C)$ urinary sodium excretion rate, and (D) urinary potassium excretion rate in FAAH WT and FAAH KO mice. C1 and C2 indicate pretreatment control infusion periods with vehicle alone; $0.25-8 \mathrm{AU}$ indicates the PIP dose in area units per 10-minute infusion; P1 and P2 indicate 10-minute post-treatment control infusion periods. (A-C) ${ }^{\#} P<0.05$ indicates a significant difference in treatment with PIP of FAAH WT vs. FAAH KO mice (for one-way and natriuretic effect of the PIP fraction was evident after either systemic (intravenous) (Figs. 5A, 6, B and C, and 7B) or local (intramedullary) administration (Fig. 5B) regardless of whether the dose was infused over 10 minutes or given as a rapid bolus. In contrast, the product was inactive as a diuretic after intraperitoneal bolus administration (5 AU; Fig. 7B). This suggests that hepatic metabolism inactivates the diuretic activity of the product. A curious aspect of the dose-response curve for the diuretic and natriuretic effects of the product after intramedullary administration was its inverse U-shape. The basis for this biphasic shape is unknown. However, it is noteworthy that cannabinoid 1 receptor agonists such as AM7418 [butyl-2-[(6aR, $10 \mathrm{a} R$ )-6a,7,10,10a-tetrahydro-1-hydroxy-9-(hydroxymethyl)6,6-dimethyl-6H-benzo[c]chromen-3-yl]-2-methylpropanoate], AM4054 [9 $\beta$-(hydroxymethyl)-3-(1-adamantyl)-hexahydrocannabinol], $\Delta$ 9-tetrahydrocannabinol, and WIN55,212-2 [(R)-(+)-[2,3dihydro-5-methyl-3-(4-morpholinylmethyl)pyrrolo[1,2,3-de]1,4-benzoxazin-6-yl]-1-naphthalenylmethanone mesylate] were shown to stimulate urine production with inverse U-shaped dose-response curves (Chopda et al., 2013). We also observed U-shaped diuresis dose-response curves when anandamide was infused into the renal medulla together with the FAAH/ monoacylglycerol lipase dual inhibitor, isopropyl dodecylfluorophosphate (Ahmad et al., 2017). One possible explanation for this effect is that tachyphylaxis, the rapid development of acute tolerance, is occurring.

In contrast to the diuretic and natriuretic effects, the pressure-lowering effect of the PIP fraction had a distinct dose response, occurring only after the higher doses tested ( $\geq 2$ AU/10 minutes) and during the post-treatment control phases (Fig. 5). The pressure-lowering effect of an intravenous infusion (1 AU/10 min) had a delayed onset, reaching significance only during the post-treatment phases, and it was prolonged, lasting even through the third post-treatment period (Fig. 6A). The finding that intraperitoneal administration of a single bolus dose of the product (5 AU) elicited a marked pressure-lowering effect of delayed onset and long duration suggests that the active substance undergoes firstpass metabolism in the liver to form a vasodepressor metabolite. Given the strong stimulation of $\mathrm{MBF}$, it seemed surprising that diuresis was not also stimulated under these conditions, as increased MBF is known to dissipate the salt and urea gradient of the inner medulla, interfering with the kidney's ability to concentrate the urine. On the other hand, it may be considered equally surprising that such a large decrease in blood pressure was not accompanied by a proportional decrease in natriuresis and diuresis, based on the pressure-natriuresis relationship. The reason for the latter may be explained by the capacity of the PIP fraction to stimulate MBF.

Our laboratory is investigating the possible relationship of the fatty acid ethanolamide system of the renal medulla to the renomedullary neutral antihypertensive lipid that Muirhead

ANOVA with Tukey post hoc tests); $* P<0.05$ indicates a significant effect of treatment with PIP vs. baseline (one-way ANOVA with the Dunnett post hoc test). (D) ${ }^{\#} P<0.05$ indicates a significant difference in treatment with PIP of FAAH WT vs. FAAH KO mice (Fisher LSD test); $* P<0.05$ indicates a significant effect of treatment with PIP vs. baseline (Fisher LSD test; $n=6$ FAAH WT and $n=7$ FAAH KO). ANOVA, analysis of variance; kwt, kidney weight; LSD, least significant difference; UV, urine formation rate. 
devoted his life to study. Published (Ritter et al., 2012) and unpublished data from our laboratory demonstrated that intramedullary infusion of anandamide stimulated diuresis and sodium and potassium excretion but had no effect on either MAP or MBF. We subsequently investigated whether similar infusion of FAAH inhibitors is able to recapitulate the effects of anandamide. The former act indirectly to cause the local accumulation of anandamide by inhibiting its hydrolysis. Intramedullary infusion of isopropyl dodecylphosphonofluoridate, a potent albeit nonselective inhibitor of FAAH, stimulated diuresis-natriuresis and enhanced $\mathrm{MBF}$, supporting that endogenous anandamide in the renal medulla can regulate renal excretory and hemodynamic functions (Ahmad et al., 2017). Because isopropyl dodecylphosphonofluoridate is also a potent inhibitor of monoacylglycerol lipase, the role of increased tissue concentrations of 2-arachidonoyl glycerol in its observed effects could not be excluded. This led us to evaluate the effects of PF-3845, a much more selective inhibitor of FAAH. Interestingly, when PF-3845 was infused into the renal medulla, diuresis-natriuresis and $\mathrm{MBF}$ were stimulated and a MAP-lowering effect was also observed (Ahmad et al., 2018). The findings reported here for the PIP fraction bear strong similarity to the effects observed after intramedullary PF-3845 in vivo. In addition, it is interesting that intramedullary PF-3845 and the PIP fraction share reported characteristics of medullipin, including the vasodepressor (Muirhead, 1993) and diuretic-natriuretic effects (Karlstrom et al., 1988). Muirhead proposed that the kidney medulla secretes a prohormone form of medullipin termed medullipin I that undergoes subsequent conversion by the hepatic cytochrome P450 system to medullipin II, the active vasodepressor form (Muirhead et al., 1988, 1989). The striking increase in vasodepressor activity after intraperitoneal administration of the PIP fraction together with its delayed onset and long-lasting nature is reminiscent of the properties ascribed to medullipin II. Further studies are needed to better define this relationship and the basis of its dependence on hepatic metabolism.

The renal medulla, through its unique cellular anatomy, blood supply, hyperosmotic environment, and interactions of interstitial cells with renal tubules and vasa recta, has long been proposed to be critical as a long-term regulator of body fluid and sodium balance and blood pressure (Zhuo, 2000). The idea that interstitial cells secrete antihypertensive lipids appeared in the literature in the late 1960 s, with morphometric observations that the lipid granules of interstitial cells decrease in experimental models of hypertension (Tobian and Ishii, 1969; Pitcock et al., 1981). The nature of these lipids has been a topic of intense research. Noted for their high content of arachidonic acid in lipid granules and the presence of high constitutive COX levels, interstitial cells were demonstrated to synthesize and secrete prostaglandin $\mathrm{E}_{2}$ in response to stimulation of receptors on their cell surface for vasopressor hormones such as angiotensin (Zusman and Keiser, 1977a). Prostaglandin $\mathrm{E}_{2}$ is a vasodilator as well as a diuretic and natriuretic (Hashimoto, 1980). To our knowledge, medullipin is the only other major antihypertensive lipid proposed to be produced and released by medullary interstitial cells (Brooks et al., 1994; Cowley, 1994).

The finding that FAAH was expressed in MMICs (Fig. 1F) provides evidence for the presence of the proposed target of PF-3845. Another finding of interest for its possible relation to secretion of the diuretic-natriuretic and vasodepressor substance was the observed increase in lipid granules in MMICs exposed to PF-3845, as demonstrated using the neutral lipid dye, Sudan Black B (Fig. 2). Ultrastructural analyses of control and PF-3845-treated MMICs suggested that the increased neutral lipid staining in the PF-3845-treated cells was associated at least in part with the increased size and number of cytoplasmic multilamellar bodies (Fig. 3). These organelles were found by us to be present in the vehicletreated MMICs (Fig. 3B, arrow) and by others in cultured medullary interstitial cells (Mathews et al., 1979) as well as in renomedullary interstitial cells in situ (Lewis and Prentice, 1979; Bohman, 1980). They are proposed to play roles in the storage and secretion of lipids (Schmitz and Muller, 1991). Further investigation is needed to determine whether multilamellar bodies are involved in the PF-3845-induced mechanism.

In summary, this study supports the blood pressure-lowering, $\mathrm{MBF}$-increasing, and diuresis/natriuresis-stimulating effects of an endogenous lipid produced by MMICs and enhanced in the presence of an FAAH inhibitor. Our data indicate a possible relationship of this compound to medullipin, the renomedullary neutral antihypertensive lipid proposed by Muirhead. Structural identification of PIP is ongoing, but preliminary data suggest that it is a sterol-related substance. Further studies are underway to identify the substance and assess its responsiveness to altered renal perfusion pressure.

\section{Authorship Contributions}

Participated in research design: Daneva, Dempsey, Ahmad, N. Li, P.-L. Li, Ritter.

Conducted experiments: Daneva, Dempsey.

Performed data analysis: Daneva, Dempsey, Ahmad, N. Li, P.-L. Li, Ritter.

Wrote or contributed to the writing of the manuscript: Daneva, Dempsey, Ahmad, N. Li, P.-L. Li, Ritter.

\section{References}

Ahmad A, Daneva Z, Li G, Dempsey SK, Li N, Poklis JL, Lichtman A, Li PL, and Ritter JK (2017) Stimulation of diuresis and natriuresis by renomedullary infusion of a dual inhibitor of fatty acid amide hydrolase and monoacylglycerol lipase. Am J Physiol Renal Physiol 313:F1068-F1076.

Ahmad A, Dempsey SK, Daneva Z, Li N, Poklis JL, Li PL, and Ritter JK (2018) Modulation of mean arterial pressure and diuresis by renomedullary infusion of a selective inhibitor of fatty acid amide hydrolase. Am J Physiol Renal Physiol 315: F967-F976.

Bermudez-Silva FJ, Viveros MP, McPartland JM, and Rodriguez de Fonseca F (2010) The endocannabinoid system, eating behavior and energy homeostasis: the end or a new beginning? Pharmacol Biochem Behav 95:375-382

Bohman SO (1980) The ultrastructure of the renal medulla and the interstitial cells, in The Renal Papilla and Hypertension (Mandal AK and Bohman SO eds) pp 7-28, Plenum, New York.

Bohman SO and Jensen PK (1976) Morphometric studies on the lipid droplets of the interstitial cells of the renal medulla in different states of diuresis. $J$ Ultrastruct Res 55:182-192.

Brooks B, Byers LW, Muirhead EE, Muirhead M, Pitcock JA, Maddipati KR, and Maxey KM (1994) Purification of class I medullipins from the venous effluent of isolated normal kidneys perfused under high pressure with saline. Blood Press 3 : 407-417.

Chopda GR, Vemuri VK, Sharma R, Thakur GA, Makriyannis A, and Paronis CA (2013) Diuretic effects of cannabinoid agonists in mice. Eur J Pharmacol 721: 64-69.

Cowley AW Jr., (1994) Franz Volhard Lecture. Evolution of the medullipin concept of blood pressure control: a tribute to Eric Muirhead. J Hypertens Suppl 12:S25-S34. Deutsch DG, Goligorsky MS, Schmid PC, Krebsbach RJ, Schmid HH, Das SK, Dey SK, Arreaza G, Thorup C, Stefano G, et al. (1997) Production and physiological actions of anandamide in the vasculature of the rat kidney. $J$ Clin Invest 100: 1538-1546.

Devane WA, Hanus L, Breuer A, Pertwee RG, Stevenson LA, Griffin G, Gibson D, Mandelbaum A, Etinger A, and Mechoulam R (1992) Isolation and structure of a brain constituent that binds to the cannabinoid receptor. Science 258:1946-1949.

DiPatrizio NV and Piomelli D (2012) The thrifty lipids: endocannabinoids and the neural control of energy conservation. Trends Neurosci 35:403-411. 
Dunn MJ, Staley RS, and Harrison M (1976) Characterization of prostaglandin production in tissue culture of rat renal medullary cells. Prostaglandins 12:37-49.

Folch J, Lees M, and Sloane Stanley GH (1957) A simple method for the isolation and purification of total lipides from animal tissues. J Biol Chem 226:497-509.

Folkow B (2007) Incretory renal functions--Tigerstedt, renin and its neglected antagonist medullipin. Acta Physiol (Oxf) 190:99-102.

Glodny B and Pauli GF (2006) The vasodepressor function of the kidney: prostaglandin E2 is not the principal vasodepressor lipid of the renal medulla. Acta Physiol (Oxf) 187:419-430.

Göthberg G, Lundin S, and Folkow B (1982) Acute vasodepressor effect in normotensive rats following extracorporal perfusion of the declipped kidney of twokidney, one clip hypertensive rats. Hypertension 4:101-105.

Hashimoto T (1980) Effects of prostaglandin E2, I2 and F2 alpha on systemic and renal hemodynamics, renal function and renin secretion in anesthetized dogs. Jpn $J$ Pharmacol 30:173-186.

He W, Xie Q, Wang Y, Chen J, Zhao M, Davis LS, Breyer MD, Gu G, and Hao C-M (2013) Generation of a tenascin-C-Cre ER2 knockin mouse line for conditional DNA recombination in renal medullary interstitial cells. PLoS ONE 8:e79839.

Ishac EJ, Jiang L, Lake KD, Varga K, Abood ME, and Kunos G (1996) Inhibition of exocytotic noradrenaline release by presynaptic cannabinoid CB1 receptors on peripheral sympathetic nerves. Br J Pharmacol 118:2023-2028.

Karlström G, Arnman V, Bergström G, Bohman SO, Rudenstam J, and Göthberg G (1990) Do angiotensin converting enzyme inhibitors lower blood pressure in the rat partly via the humorally mediated antihypertensive system of the renal medulla? J Hypertens 8:501-513.

Karlström G, Arnman V, Folkow B, and Göthberg G (1988) Activation of the humoral antihypertensive system of the kidney increases diuresis. Hypertension 11 $597-601$.

Lake KD, Martin BR, Kunos G, and Varga K (1997) Cardiovascular effects of anandamide in anesthetized and conscious normotensive and hypertensive rats. Hypertension 29:1204-1210.

Lemley KV and Kriz W (1991) Anatomy of the renal interstitium. Kidney Int 39 $370-381$.

Lewis DJ and Prentice DE (1979) Ultrastructure of rhesus monkey renomedullary interstitial cells. Lab Anim 13:75-79.

Li J and Wang DH (2006) Differential mechanisms mediating depressor and diuretic effects of anandamide. $J$ Hypertens 24:2271-2276.

Li N, Yi F, Sundy CM, Chen L, Hilliker ML, Donley DK, Muldoon DB, and Li PL (2007) Expression and actions of HIF prolyl-4-hydroxylase in the rat kidneys. Am J Physiol Renal Physiol 292:F207-F216.

Li N, Zhang G, Yi FX, Zou AP, and Li PL (2005) Activation of NAD(P)H oxidase by outward movements of $\mathrm{H}+$ ions in renal medullary thick ascending limb of Henle. Am J Physiol Renal Physiol 289:F1048-F1056.

Long JZ, LaCava M, Jin X, and Cravatt BF (2011) An anatomical and temporal portrait of physiological substrates for fatty acid amide hydrolase. J Lipid Res 52: 337-344.

Mathews J, DuCharme DW, and McCandlis M (1979) Morphology of cultured renomedullary interstitial cells. Micron 10:185-186.

Moeckel GW, Zhang L, Fogo AB, Hao CM, Pozzi A, and Breyer MD (2003) COX2 activity promotes organic osmolyte accumulation and adaptation of renal medullary interstitial cells to hypertonic stress. J Biol Chem 278:19352-19357.
Muirhead EE (1993) Renal vasodepressor mechanisms: the medullipin system. $J$ Hypertens Suppl 11:S53-S58.

Muirhead EE, Brooks B, Byers LW, Pitcock JA, and Brown PS (1988) Conversion of medullipin I by the liver. Trans Assoc Am Physicians 101:226-234.

Muirhead EE, Byers LW, Capdevila J, Brooks B, Pitcock JA, and Brown PS (1989) The renal antihypertensive endocrine function: its relation to cytochrome P-450. Hypertens 7:361-369.

Muirhead EE, Rightsel WA, Pitcock JA, and Inagami T (1990) Isolation and culture of juxtaglomerular and renomedullary interstitial cells. Methods Enzymol 191:152-167.

Pitcock JA, Brown PS, Byers W, Brooks B, and Muirhead EE (1981) Degranulation of renomedullary interstitial cells during reversal of hypertension. Hypertension 3:II75-II-80.

Ritter JK, Li C, Xia M, Poklis JL, Lichtman AH, Abdullah RA, Dewey WL, and Li PL (2012) Production and actions of the anandamide metabolite prostamide E2 in the renal medulla. J Pharmacol Exp Ther 342:770-779.

Schmitz G and Müller G (1991) Structure and function of lamellar bodies, lipidprotein complexes involved in storage and secretion of cellular lipids. J Lipid Res 32:1539-1570

Schneider JP, Pedersen L, Mühlfeld C, and Ochs M (2015) Staining histological lung sections with Sudan Black B or Sudan III for automated identification of alveolar epithelial type II cells. Acta Histochem 117:675-680.

Tobian L and Ishii M (1969) Interstitial cell granules and solutes in renal papilla in post-Goldblatt hypertension. Am J Physiol 217:1699-1702.

Wang Z, Tang L, Zhu Q, Yi F, Zhang F, Li PL, and Li N (2011) Hypoxia-inducible factor- $1 \alpha$ contributes to the profibrotic action of angiotensin II in renal medullary interstitial cells. Kidney Int 79:300-310.

Wise LE, Cannavacciulo R, Cravatt BF, Martin BF, and Lichtman AH (2008) Evaluation of fatty acid amides in the carrageenan-induced paw edema model. Neuropharmacology 54:181-188.

Zhu Q, Xia M, Wang Z, Li PL, and Li N (2011) A novel lipid natriuretic factor in the renal medulla: sphingosine-1-phosphate. Am J Physiol Renal Physiol 301: F35-F41.

Zhuo JL (2000) Renomedullary interstitial cells: a target for endocrine and paracrine actions of vasoactive peptides in the renal medulla. Clin Exp Pharmacol Physiol 27:465-473.

Zou AP, Yang ZZ, Li PL, and Cowley AW JR. (2001) Oxygen-dependent expression of hypoxia-inducible factor-1alpha in renal medullary cells of rats. Physiol Genomics 6:159-168.

Zusman RM and Keiser HR (1977a) Prostaglandin biosynthesis by rabbit renomedullary interstitial cells in tissue culture. Stimulation by angiotensin II, bradykinin, and arginine vasopressin. J Clin Invest 60:215-223.

Zusman RM and Keiser HR (1977b) Prostaglandin E2 biosynthesis by rabbit renomedullary interstitial cells in tissue culture. Mechanism of stimulation by angiotensin II, bradykinin, and arginine vasopressin. J Biol Chem 252:2069-2071.

Address correspondence to: Dr. Joseph K. Ritter, Department of Pharmacology and Toxicology, Virginia Commonwealth University School of Medicine 1217 E. Marshall St., Kontos Medical Sciences Building, Room 530, Box 980613, Richmond, VA 23298-0613. E-mail: jritter@vcu.edu 